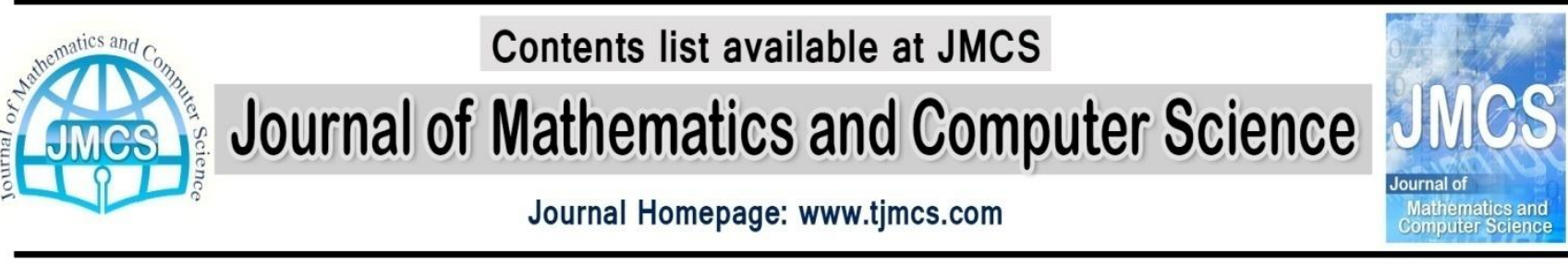

\section{Sigma Ideal Amenability of Banach Algebras}

\author{
M. Momeni ${ }^{1}$, T. Yazdanpanah ${ }^{2}$, M. R. Mardanbeigi ${ }^{3}$ \\ ${ }^{1}$ Department of Mathematics, Science and Research Branch, Islamic Azad University (IAU), Tehran \\ 1477893855, Iran \\ E-mail address: srb.maryam@gmail.com \\ ${ }^{2}$ Department of Mathematics, Persian Gulf University, Bushehr 75169, Iran \\ E-mail address: yazdanpanah@pgu.ac.ir \\ ${ }^{3}$ Department of Mathematics, Science and Research Branch, Islamic Azad University (IAU), Tehran \\ 1477893855, Iran \\ E-mail address: $\underline{\text { mrmardanbeigi@ srbiau.ac.ir }}$
}

Article history:

Received August 2013

Accepted September 2013

Available online October 2013

\begin{abstract}
Let $A$ be a Banach algebra and let $I$ be a closed two-sided ideal in $A$. $A$ is $I$-weakly amenable if $H^{l}\left(A, I^{*}\right)=\{0\}$. Further, $\mathrm{A}$ is ideally amenable if $A$ is $I$-weakly amenable for every closed twosided ideal $I$ in $A$. In this paper we introduce $\sigma$-ideal amenability for a Banach algebra $A$, where $\sigma$ is an idempotent bounded endomorphism of $A$.
\end{abstract}

Key words: $\sigma$-ideally amenable, $\sigma$-weakly amenable, closed two-sided ideal, $\sigma$-derivation. 2010 Mathematics Subject Classification: $46 \mathrm{H} 25$.

\section{Introduction}

Let $A$ be a Banach algebra and let $X$ be a Banach $A$-bimodule. Then $X^{*}$, the dual space of $X$, with the following module actions is a Banach $A$-bimodule,

$$
\left\langle x, a \cdot x^{*}\right\rangle=\left\langle x \cdot a, x^{*}\right\rangle, \quad\left\langle x, x^{*} \cdot a\right\rangle=\left\langle a \cdot x, x^{*}\right\rangle, \quad\left(a \in A, x \in X, x^{*} \in X^{*}\right) .
$$

In particular, if $I$ is a closed ideal in $A$, then $I$ and $I^{*}$ will be a Banach $A$-bimodule and a dual Banach $A$-bimodule respectively.

Suppose that $A$ is a Banach algebra and $X$ is a Banach $A$-bimodule. A linear map $D: A \rightarrow X$ is called a derivation if

$$
D(a b)=a D(b)+D(a) b, \quad(a, b \in A) .
$$

Given $x \in X$, the map $\delta_{x}(a)=a x-x a$ is a derivation on $A$ which is called an inner derivation. The set of all bounded derivations and inner derivations are denoted by $Z^{1}(A, X)$ and $N^{1}(A, X)$, respectively. $H^{1}(A, X)=Z^{1}(A, X) / N^{1}(A, X)$ is called the first cohomology of $A$ with coefficients in $X$.

A Banach algebra $A$ is called amenable if $H^{1}\left(A, X^{*}\right)=\{0\}$ for every Banach $A$-bimodule $X$. 
Let $A$ be a Banach algebra and $\sigma$ be a bounded endomorphism of $A$, i.e. a bounded Banach algebra homomorphism from $A$ into $A$. A $\sigma$-derivation from $A$ into a Banach $A$-bimodule $X$ is a bounded linear map $D: A \rightarrow X$ satisfying

$$
D(a b)=\sigma(a) \cdot D(b)+D(a) \cdot \sigma(b), \quad(a, b \in A) .
$$

For each $x \in X$, the mapping

$$
\delta_{x}^{\sigma}: A \rightarrow X
$$

definedby $\delta_{x}^{\sigma}(a)=\sigma(a) \cdot x-x \cdot \sigma(a)$, for all $a \in A$, is a $\sigma$-derivation called an inner $\sigma$ derivation.

Let $A$ be a Banach algebra and $I$ be a closed two-sided ideal in $A$. Then $\mathrm{A}$ is called $I$-weakly amenable if $H^{1}\left(A, I^{*}\right)=\{0\}$. Furthermore $A$ is called ideally amenable if $H^{1}\left(A, I^{*}\right)=\{0\}$, for every closed twosided ideal $I$ of $A$. This definitions was introduced by Eshaghi Gordji and Yazdanpanah in [1]. In this paper we introduce $\sigma$ - $I$-weakly amenability and $\sigma$-ideal amenability of Banach algebras.

\section{2. $\sigma$-ideal amenability}

We start this section with the following definition which is the basic definition for the present paper.

Definition 1 Let $A$ be a Banach algebra and $\sigma$ be a bounded endomorphism of $A$. Let $I$ be a closed two-sided ideal of $A$, then $A$ is said to be $\sigma$ - $I$-weakly amenable if every $\sigma$-derivation from $A$ into $I^{*}$ is $\sigma$-inner. Furthermore $A$ is $\sigma$-ideally amenable if $A$ is $\sigma-I$-weakly amenable for every closed twosided ideal $I$ of $A$.

Proposition 2 Let $A$ be a Banach algebra and $\sigma$ be an idempotent epimorphism of $A$. If $A$ is $\sigma$ weakly amenable, then $A$ is essential.

Proof. Assume towards a contradiction that $\overline{A^{2}} \neq A$. So there exists $b_{0} \in A$ such that $b_{0} \notin \overline{A^{2}}$. By Hahn-Banach theorem there exists $a_{0}^{*} \in A^{*}$ with $a_{0}^{*} \mid A^{2}=0$ and $\left\langle b_{0}, a_{0}^{*}\right\rangle=1$. Since $\sigma$ is surjective, there exists $a_{0} \in A$ such that $b_{0}=\sigma\left(a_{0}\right)$ and so $\left\langle\sigma\left(a_{0}\right), a_{0}^{*}\right\rangle=1$ Define $D: A \rightarrow A^{*}$ with

$$
\langle b, D(a)\rangle=\left\langle\sigma(a), a_{0}^{*}\right\rangle\left\langle\sigma(b), a_{0}^{*}\right\rangle, \quad(a, b \in A)
$$

So $D$ is a continuous linear map. We show that $D$ is a $\sigma$-derivation. First note $\sigma$ is an epimorphism and $a_{0}^{*} \mid A^{2}=0$ so $a_{0}^{*} \mid \sigma\left(A^{2}\right)=0$. Thus for each $a, b \in A$, we have

$$
\langle c, D(a b)\rangle=\left\langle\sigma(a b), a_{0}^{*}\right\rangle\left\langle\sigma(c), a_{0}^{*}\right\rangle=0, \quad(c \in A) .
$$

On the other hand for each $c \in A$

$$
\begin{aligned}
\langle c, \sigma(a) \cdot D(b)\rangle+\langle c, D(a) \cdot \sigma(a b)\rangle & =\langle c \sigma(a), D(b)\rangle+\langle\sigma(b) c, D(a)\rangle \\
& =\left\langle\sigma(c \sigma(a)), a_{0}^{*}\right\rangle\left\langle\sigma(b), a_{0}^{*}\right\rangle \\
& +\left\langle\sigma(\sigma(b) c), a_{0}^{*}\right\rangle\left\langle\sigma(a), a_{0}^{*}\right\rangle \\
& \left.=\langle\sigma(c) \sigma(a)), a_{0}^{*}\right\rangle\left\langle\sigma(b), a_{0}^{*}\right\rangle \\
& +\left\langle\sigma(b) \sigma(c), a_{0}^{*}\right\rangle\left\langle\sigma(a), a_{0}^{*}\right\rangle \\
& =0,
\end{aligned}
$$

because $a_{0}^{*} \mid \sigma\left(A^{2}\right)=0$. Thus $D$ is a $\sigma$-derivation. Now

$$
\begin{aligned}
\left\langle\sigma\left(a_{0}\right), D\left(a_{0}\right)\right\rangle & =\left\langle\sigma\left(a_{0}\right), D\left(a_{0}\right)\right\rangle=\left\langle\sigma\left(\sigma\left(a_{0}\right)\right), a_{0}^{*}\right\rangle\left\langle\sigma\left(a_{0}\right), a_{0}^{*}\right\rangle \\
& =\left\langle\sigma\left(a_{0}\right), a_{0}^{*}\right\rangle\left\langle\sigma\left(a_{0}\right), a_{0}^{*}\right\rangle \\
& =1 .
\end{aligned}
$$

But

$$
\begin{aligned}
\left\langle\sigma\left(a_{0}\right), \delta_{a^{*}}^{\sigma}\left(a_{0}\right)\right\rangle & =\left\langle\sigma\left(a_{0}\right), \sigma\left(a_{0}\right) \cdot a^{*}-a^{*} \cdot \sigma\left(a_{0}\right)\right\rangle \\
& =\left\langle\sigma\left(a_{0}\right) \sigma\left(a_{0}\right), a^{*}\right\rangle-\left\langle\sigma\left(a_{0}\right) \sigma\left(a_{0}\right), a^{*}\right\rangle \\
& =0 \quad\left(a^{*} \in A^{*}\right)
\end{aligned}
$$

So $D$ is not $\sigma$-inner. and it is a contradiction of the fact that $A$ is $\sigma$-weakly amenable.

Remark 3 Let $A$ be a non-unital Banach algebra. We denote by $A^{\#}$ the Banach algebra formed by adjoining an identity to $A$, so that $A^{\#}=A \oplus C e$, with the product 


$$
(a, \alpha)(b, \beta)=(a b+\alpha b+\beta a, \alpha \beta) \quad(\alpha, \beta \in \mathbb{C}, a, b \in A)
$$

Let $A$ be a non-unital Banach algebra with adjoined identity $e$, take $e^{\prime} \in\left(A^{\#}\right)^{*}$ with $\langle e, e ́(\rangle=1$ and $e ́ \mid A=0$, and extend $a^{*} \in A^{*}$ to an element of $\left(A^{\#}\right)^{*}$ by setting $\left(e, a^{*}\right)=0$. Then $\left(A^{\#}\right)^{*}=A^{*} \oplus \mathbb{C} e ́$ as a Banach space, and $\left(A^{\#}\right)^{*}$ is an $A^{\#}$-bimodule with the following module actions,

$$
\begin{aligned}
& (a+\alpha e) \cdot\left(a^{*}+\beta \dot{e}\right)=a a^{*}+\alpha a^{*}+\left(\left\langle a, a^{*}\right\rangle+\alpha \beta\right) \dot{e}, \\
& \left(a^{*}+\beta \dot{e}\right) \cdot(a+\alpha e)=a^{*} a+\alpha a^{*}+\left(\left\langle a, a^{*}\right\rangle+\alpha \beta\right) \dot{e} .
\end{aligned}
$$

Proposition 4 Let $A$ be a Banach algebra and $\sigma$ be an idempotent epimorphism of $A$. If $A$ is $\sigma$ weakly amenable, then $A^{\#}$ is $\hat{\sigma}$-weakly amenable, where $\hat{\sigma}$ is the endomorphism of $A^{\#}$ induced by $\sigma$, i.e. $\hat{\sigma}(a+\alpha)=\sigma(a)+\alpha$.

Proof. Let $D: A^{\#} \rightarrow\left(A^{\#}\right)^{*}$ be a $\hat{\sigma}$-derivation. Since $D(e)=0$ we can look at $D$ as a map from $A$ into $\left(A^{\#}\right)^{*}$. Also since $\left(A^{\#}\right)^{*}=A^{*} \oplus \mathbb{C} e$, there exists two bounded linear maps $\lambda: A \rightarrow \mathbb{C}$ and $d: A \rightarrow A^{*}$ such that $D(a)=d(a)+\lambda(a) e ́,(a \in A)$. Since $\hat{\sigma} \mid A=\sigma$, by previose Remark for each $a, b \in A$ we have,

$$
\begin{aligned}
d(a b)+\lambda(a b) & =D(a b) \\
& =\sigma(a) \cdot D(b)+D(a) \cdot \sigma(b) \\
& =\sigma(a) \cdot[d(b)+\lambda(b)]+[d(a)+\lambda(a)] \cdot \sigma(b) \\
& =\sigma(a) d(b)+\langle\sigma(a), d(b)\rangle+d(a) \sigma(b) \\
& +\langle\sigma(b), d(a)\rangle .
\end{aligned}
$$

So

$$
d(a)=\sigma(a) \cdot a^{*}+a^{*} \cdot \sigma(a), \quad(a \in A) .
$$

and

$$
\lambda(a b)=\langle\sigma(a), d(b)\rangle+\langle\sigma(b), d(a)\rangle .
$$

Therefore $d: A \rightarrow A^{*}$ is a $\sigma$-derivation. So there exists $a^{*} \in A^{*}$ such that

$$
d(a)=\sigma(a) \cdot a^{*}+a^{*} \cdot \sigma(a) \quad(a \in A) .
$$

Now we show that $\lambda \mid A^{2}=0$. let $a, b \in A$, we have,

$$
\begin{aligned}
\langle\sigma(a) \sigma(b), \lambda\rangle & =\langle\sigma(\sigma(a)), d(\sigma(b))\rangle+\langle\sigma(\sigma(b)), d(\sigma(a))\rangle \\
& =\left\langle\sigma(a), \sigma(b) \cdot a^{*}-a^{*} \cdot \sigma(b)\right\rangle+\left\langle\sigma(b), \sigma(a) \cdot a^{*}-a^{*} \cdot \sigma(a)\right\rangle \\
& =0
\end{aligned}
$$

Which shows that $\lambda \mid \sigma\left(A^{2}\right)=0$. Since $\sigma$ is an epimorphism, so $\lambda \mid A^{2}=0$. Also since $A$ is $\sigma$ weakly amenable by Proposition $2,\left(\overline{A^{2}}\right)=A$. Thus $\lambda=0$ on $A$. Therefore $D=d$ is a $\sigma$ inner derivation.

Proposition 5 Let $\sigma$ be a bounded endomorphism of Banach algebra $A$. If $A^{\#}$ is $\hat{\sigma}$-weakly amenable, Then $A$ is $\sigma$-weakly amenable.

Proof. Let $D: A \rightarrow A^{*}$ be a continuous $\hat{\sigma}$-derivation. Note $A$ is a Banach $A^{\#}$-bimodule with the following module actions:

$$
(a+\alpha) \cdot b=a \cdot b+\alpha b, \quad b \cdot(a+\alpha)=b \cdot a+\alpha b,
$$

for all $a, b \in A, \alpha \in \mathbb{C}$. Define $\widetilde{D}: A^{\#} \rightarrow A^{*}$ with $\widetilde{D}(a+\alpha)=D(a)$. Clearly $\widetilde{D}$ is continuous $\sigma$ derivation and we can look at it as a function into $\left(A^{\#}\right)^{*}$.

Since $A^{\#}$ is $\hat{\sigma}$-weakly amenable, so there exists $a^{*} \in A^{*}$ such that $\widetilde{D}=\delta_{a^{*}}^{\sigma}$. Hence for each a $A$ we have

$$
D(a)=\widetilde{D}(a+\alpha)=\hat{\sigma}(a+\alpha) a^{*}-a^{*} \hat{\alpha}=\sigma(a) a^{*}-a^{*} \sigma(a) .
$$


Which shows that $D$ is $\sigma$-inner and so $A$ is $\sigma$-weakly amenable.

Proposition 6 Let $A$ be a Banach algebra and $I$ be a closed two-sided ideal of $A$ with a bounded approximate identity. Let $\sigma$ be an idempotent endomorphism of $A$ such that $\sigma(I)=I$. Then $I$ is $\sigma$ weakly amenable if and only if $H_{\sigma}^{1}\left(A, I^{*}\right)=\{0\}$.

Proof. Suppose that $I$ is $\sigma$-weakly amenable and let $D: A \rightarrow I^{*}$ be a $\sigma$-derivation and $i: I \rightarrow A$ be the embeding map. Then $d=\left.D\right|_{I}: I \rightarrow I^{*}$ is a $\sigma$-derivation. So there exists $i^{*} \in I^{*}$ such that $d=\delta_{i^{*}}^{\sigma}$. Since $I$ is $\sigma$-weakly amenable and $\sigma(I)=I$ by Proposition $2, \overline{\sigma\left(I^{2}\right)}=\overline{I^{2}}=I$.On the other hand for $i$. $j \in I$ we have,

Therefore $D=\delta_{i^{*}}^{\sigma}$, and so $D$ is $\sigma$-inner.

$$
\begin{aligned}
\langle\sigma(i j), D(a)\rangle & =\langle\sigma(i) \sigma(j), D(a)\rangle \\
& =\langle\sigma(i) \sigma(j), D(a)\rangle \\
& =\langle\sigma(i) D(j a)-D(j) \sigma(a)\rangle \\
& =\left\langle\sigma(i), \sigma(j a) \cdot i^{*}-i^{*} \cdot \sigma(j a)\right\rangle \\
& -\left\langle\sigma(a) \sigma(i), \sigma(j) \cdot i^{*}-i^{*} \cdot \sigma(j)\right\rangle \\
& =\left\langle\sigma(i j), \sigma(a) i^{*}\right\rangle-\left\langle\sigma(i j), i^{*} \sigma(a)\right\rangle \\
& =\left\langle\sigma(i j), \delta_{i^{*}}^{\sigma}(a)\right\rangle \quad(a \in A) .
\end{aligned}
$$

Conversely, let $H_{\sigma}^{1}\left(A, I^{*}\right)=\{0\}$. and let $D: I \rightarrow I^{*}$ be a $\sigma$-derivation. Since $I$ is $\sigma$-neo-unital Banach $I$-bimodule, i.e. $I=\sigma(I) \cdot I \cdot \sigma(I)$, by [2, Proposition 37], $D$ has an extension $\widetilde{D}: A \rightarrow I^{*}$ such that $\widetilde{D}$ is also $\sigma$-derivation. Now by hypothesis $\widetilde{D}$ is $\sigma$-inner. Thus $I$ is $\sigma$-weakly amenable.

Proposition 7 Let $\sigma$ be a bounded endomorphism of Banach algebra $A$. If $A^{\#}$ is $\hat{\sigma}$-ideally amenable, then $A$ is $\sigma$-ideally amenable.

Proof. Let $I$ be a closed two-sided ideal of $A$ and $D: A \rightarrow I$ be a $\sigma$-derivation. It is easy to see that $I$ is a closed two-sided ideal of $A^{\#}$, and $\widetilde{D}: A^{\#} \rightarrow I^{*}$ with $D^{\#}(a+\alpha)=D(a), \quad(a \in A, \alpha \in \mathbb{C})$ is a $\hat{\sigma}$ derivation. Hence there exists $i^{*} \in I^{*}$ such that $D^{\#}=\delta_{i^{*}}^{\sigma}$. So for each $a \in A$ we have

$$
D(a)=\widetilde{D}(a+\alpha)=\hat{\sigma}(a+\alpha) \cdot i^{*}-i^{*} \cdot \hat{\sigma}(a+\alpha)=\sigma(a) \cdot i^{*}-i^{*} \cdot \sigma(a)
$$

Which shows that $D$ is $\sigma$-inner. So $A$ is $\sigma$-ideally amenable.

Proposition 8 Let $A$ be a Banach algebra and $\sigma$ be an idempotent epimorphism of $A$. Suppose $A$ is $\sigma$ weakly amenable and for each closed two-sided ideal $I$ such that $I=\overline{A I U I A}, A$ is $\sigma-I$ weakly amenable. Then $A$ is $\sigma$-ideally amenable.

Proof. Let $I$ be a closed two-sided ideal in $A$. Put $J=\overline{A I U I A}$. It is easy to see that $J$ is a closed twosided ideal in $A$ and $J=\overline{A J U J A}$. Let $l: J \rightarrow I$ be the natural embeding and $D: A \rightarrow I^{*}$ be a $\sigma$ derivation. Then $\iota^{*} \circ D: A \rightarrow J^{*}$ is a derivation. So there exists $m \in J^{*}$ such that $\iota^{*} \circ D=\delta_{m}^{\sigma}$. Let $x^{*}$ be the extension of $m$ into $I$, by the Hahn-Banach theorem, for every $a, b \in A$ we have,

$$
\begin{aligned}
\langle i, D(a b)\rangle & =\langle i \sigma(a), D(b)\rangle+\langle\sigma(b) i, D(a)\rangle \\
& =\langle\iota(i \sigma(a)), D(b)\rangle+\langle\imath(\sigma(b) i), D(a)\rangle \\
& =\left\langle i \sigma(a), \iota^{*} \circ D(b)\right\rangle+\left\langle\sigma(b) i, \iota^{*} \circ D(a)\right\rangle \\
& =\langle i \sigma(a), \sigma(b) m-m \sigma(b)\rangle+\langle\sigma(b) i, \sigma(a) m-m \sigma(a)\rangle \\
& =\langle i, \sigma(a b) m-m \sigma(a b)\rangle \\
& =\left\langle i, \delta_{x^{*}}^{\sigma}(a b)\right\rangle
\end{aligned}
$$

Hence $D(a b)=\delta_{x^{*}}^{\sigma}(a b)$. Since $A$ is $\sigma$-weakly amenable, so $\left(\overline{A^{2}}\right)=A$ and therefore $D=\delta_{x^{*}}^{\sigma}$ and $D$ is $\sigma$-inner.

Proposition 9 Let $A$ be a Banach algebra and $\sigma$ be a bounded idempotent endomorphism of $A$. Let $I$ be a closed two-sided ideal in $A$ with a bounded approximate identity. If $A$ is $\sigma$-ideally amenable, then $I$ is $\sigma$-ideally amenable.

Proof. Let $J$ be a closed two-sided ideal in $I$. It is easy to see that $J$ is an ideal in $A$. Let $D: I \rightarrow J^{*}$ be a $\sigma$-derivation. By [2, proposition 37], $D$ can be extended to a $\sigma$-derivation, $\widetilde{D}: A \rightarrow J^{*}$. So there exists $m \in J^{*}$ such that $D=\delta_{x^{*}}^{\sigma}$. Thus $D(i)=\widetilde{D}(i)=\delta_{x^{*}}^{\sigma}$, for each $i \in I$. So $D$ is $\sigma$-inner. 
Definition 10 Let $I$ be a closed two-sided ideal in Banach algebra $A$. We say that $I$ has the trace extension property, if every $m \in I^{*}$ such that $a m=m a$ for each $a \in A$, can be extended to $a^{*} \in A^{*}$ such that $a a^{*}=a^{*} a$ for each $a \in A$.

Proposition 11 Let $A$ be a Banach algebra and $\sigma$ be a bounded endomorphism of $A$ with dense range. suppose $A$ is a $\sigma$-ideally amenable and $I$ be a closed two-sided ideal in $A$ that has the trace extension property. Then $\frac{A}{I}$ is $\sigma$-ideally amenable.

Proof. Let $\frac{J}{I}$ be a closed two-sided ideal in $\frac{A}{I}$. Then $J$ is a closed two-sided ideal in $A$. Suppose $\pi: J \rightarrow$ $\frac{J}{I}$ and $q: A \rightarrow \frac{A}{I}$ are natural quotient maps and $\pi^{*}$ is adjoint of $\pi$. Let $D: \frac{A}{I} \rightarrow\left(\frac{J}{I}\right)^{*}$ be a $\sigma$-derivaton. Then $\pi^{*} \circ D \circ q: A \rightarrow J^{*}$ is a $\sigma$-derivation. So there exists $x^{*} \in J^{*}$ such that $\pi^{*} \circ D \circ q=\delta_{x^{*}}^{\sigma}$. Let $m$ be the restriction of $x^{*}$ to $I$. Then $m \in I^{*}$ and for each $i \in I$ we have,

$$
\begin{array}{rlr}
\langle i, \sigma(b) m-m \sigma\rangle & =\langle i \sigma(b)-\sigma(b) i, m\rangle & \\
& =\left\langle i \sigma(b)-\sigma(b) i, x^{*}\right\rangle & \\
& =\left\langle i, \sigma(b) x^{*}-x^{*} \sigma(b)\right\rangle & \\
& =\left\langle i, \delta_{x^{*}}^{\sigma}\right\rangle=\left\langle i, \pi^{*} \circ D \circ q(b)\right\rangle \\
& =\langle\pi(i), D \circ q(b)\rangle \\
& =\langle I, D(b+I)\rangle=0 \quad(b \in A)
\end{array}
$$

Therefore $\sigma(b) m=m \sigma(b)$ for each $b \in A$. Since $\sigma$ has a dense range, for each $a \in A$, there exists a net $\left(b_{\alpha}\right) \subseteq A$, such that $a=\lim _{\alpha} \sigma\left(b_{\alpha}\right)$. So for each $a \in A$ we have,

$$
a m=\lim \sigma\left(b_{\alpha}\right) m=\lim m \sigma\left(b_{\alpha}\right) m=m l i m g\left(b_{\alpha}\right) m=m a
$$

Now since $I$ has the trace êxtension propêrty, $m$ can be exteñ ${ }^{\alpha}$ to $a^{*} \in A^{*}$ such that $a a^{*}=a^{*} a$, for each $a \in A$. Let $y^{*}$ be the restriction of $a^{*}$ to $J$. Then $y^{*} \in J^{*}$ and $x^{*}-y^{*}=0$ on $I$. Therefore $x^{*}-y^{*} \in\left(\frac{J}{I}\right)^{*}$ and we have,

$$
\begin{aligned}
\langle j+I, D(a+I)\rangle & =\langle\pi(j), D(q(a))\rangle \\
& =\left\langle j, \pi^{* \circ} D^{\circ} q(a)\right\rangle \\
& =\left\langle j, \delta_{x^{*}}^{\sigma}(a)\right\rangle \\
& =\left\langle j, \delta_{x^{*}-y^{*}}^{\sigma}(a)\right\rangle \\
& =\left\langle j+I, \delta_{x^{*}-y^{*}}^{\sigma}(a+I)\right\rangle \quad(j \in J)
\end{aligned}
$$

Hence $D=\delta_{x^{*}-y^{*}}^{\sigma}$ and therefore $\frac{A}{I}$ is $\sigma$-ideally amenable.

Proposition 12 Let $A$ be a Banach algebra and let $j$ be a closed two-sided ideal in $A$ with a bounded approximate identity. Let $\sigma$ be a bounded idempotent endomorphism of $A$. Then for every closed twosided ideal $I$ in $J, J$ is $\sigma$-I- weakly amenable if and only if $A$ is $\sigma$ - $I$-weakly amenable.

Proof. Let $\left(e_{\alpha}\right)_{\alpha \in I}$ be a bounded approximate identity for $J$ and $D: J \rightarrow I^{*}$ be a $\sigma$-derivation. In [2, Proposition 37], we showed that the map $\widetilde{D}: A \rightarrow I^{*}$ defined by

$$
\widetilde{D}(a)=w^{*}-\lim _{\alpha}\left(D\left(a e_{\alpha}\right)\right) \quad(a \in A)
$$

is a countinuous $\sigma$-derivation. If $D=0$ then $\widetilde{D}=0$, since $J I=I J=I$. Therefore $H_{\sigma}^{1}\left(J, I^{*}\right)=$ $H_{\sigma}^{1}\left(A, I^{*}\right)$ and this implies that $A$ is $\sigma-I$-weakly amenable if and only if $J$ is $\sigma-I$-weakly amenable.

Proposition 13 Let $A$ be a Banach algebra whit a bounded approximate identity and $\sigma$ be a bounded idempotent epimorphism of $A$. Let $\varphi$ be a non-trivial character on $A$ and $I=\operatorname{ker} \varphi$. If $\sigma(I) \subseteq I$, then

1) Every $\sigma$-derivation $D: A \rightarrow A^{*}$ restricts to a $\sigma$-derivation $d: I \rightarrow I^{*}$ such that there exists a function $\psi: I \rightarrow \mathbb{C}$ satisfying

$$
\psi(i j)=\langle\sigma(i), D(j)\rangle+\langle\sigma(j), D(i)\rangle \quad(i, j \epsilon I)
$$

2) Every $\sigma$-derivation $D: I \rightarrow I^{*}$ for which there exists such a function $\psi$ extends to a $\sigma$-derivation $\widetilde{D}: A \rightarrow A^{*}$.

Proof. 1) Clearly every $\sigma$-derivation restricts to a $\sigma$-derivation. Let $\left(\mathrm{e}_{\alpha}\right)$ be a bounded approximate identity in $A$. So the bounded net $\left(\widehat{e_{\alpha}}\right) \subseteq A^{* *}$ with $\left\langle a^{*}, \widehat{e_{\alpha}}\right\rangle=\left\langle e_{\alpha}, a^{*}\right\rangle$, $\left(a^{*} \in A^{*}\right)$ has a weak*convergence subnet. Thus without loss of generality, we may suppose that for each $a \in A^{*}$, the limit of the net $\left\langle e_{\alpha}, a^{*}\right\rangle$ exists. Now define $\psi(i)=\lim _{\alpha}\left\langle e_{\alpha}, D(i)\right\rangle$ then for each $i, j \in I$ we have,

$$
\psi(i j)=\lim _{\alpha}\left\langle e_{\alpha}, D(i j)\right\rangle
$$




$$
\begin{aligned}
& =\lim _{\alpha}\left\langle e_{\alpha}, \sigma(i) D(j)+D(i) \sigma(j)\right\rangle \\
& =\lim _{\alpha}\left\langle e_{\alpha} \sigma(i), D(j)\right\rangle+\langle D(i) \sigma(j)\rangle \\
& =\lim _{\alpha}\left\langle e_{\alpha} \sigma(i), D(j)\right\rangle+\left\langle\sigma(i) e_{\alpha}, D(i)\right\rangle \\
& =\langle\sigma(i), D(j)\rangle+\langle\sigma(j), D(j)\rangle
\end{aligned}
$$

2)Let $\left(e_{\alpha}\right)$ be a bounded pproximate identity for $A$. Then $\lim _{\alpha} \varphi\left(e_{\alpha}\right)=1$. Hence we may assume that $\varphi\left(e_{\alpha}\right)=1$ for all $\alpha$. Let $D: I \rightarrow I^{*}$ be a $\sigma$-derivation. Since for each $a \in A, a-\varphi(a) e_{\alpha} \in I$. So for each $i \in I$ every extension of $D(i) \in I$ to $A^{*}$ is unique. Therefore we can extend $D$ to $D_{1}: I \rightarrow A^{*}$ with

$$
\left\langle a, D_{1}(i)\right\rangle=\lim _{\alpha}\left\langle a-\varphi(a) e_{\alpha}, D(i)\right\rangle+\varphi(a) \psi(i)
$$

We show that $D_{1}$ is a $\sigma$-derivation from ${ }^{\alpha} I$ to $A^{*}$.

$$
\begin{aligned}
\left\langle a, D_{1}(i j)\right\rangle & =\lim _{\alpha}\left\langle a-\varphi(a) e_{\alpha}, D(i j)\right\rangle+\varphi(a) \psi(i j) \\
& =\lim _{\alpha}\left\langle a-\varphi(a) e_{\alpha}, D(i) \sigma(j)+\sigma(i) D(j)\right\rangle+\varphi(a) \psi(i j) \\
& =\lim _{\alpha}\left\langle\sigma(j)\left(a-\varphi(a) e_{\alpha}, D(i)\right\rangle\right. \\
& +\left\langle a-\varphi(a) e_{\alpha}, \sigma(i), D(j)\right\rangle+\varphi(a) \psi(i j) \\
& =\langle\sigma(j) a-\sigma(j) \varphi(a), D(i)\rangle \\
& +\langle a \sigma(i)-\varphi(a) \sigma(i), D(j)\rangle \\
& +\varphi(a)(\langle\sigma(i), D(j)\rangle)+\langle\sigma(j), D(i)\rangle \\
& =\langle\sigma(j) a, D(i)\rangle+\langle a \sigma(i), D(j)\rangle \\
& =\lim _{\alpha}\left\langle\sigma(j) a-\varphi(\sigma(j) a) e_{\alpha}, D(i)\right\rangle \\
& =\lim _{\alpha}\left\langle a \sigma(i)-\varphi(a \sigma(i)) e_{\alpha}, D(j)\right\rangle \\
& +\varphi(\sigma(j) a) \psi(i)+\varphi(a \sigma(i) \psi(j) \\
& =\left\langle\sigma(j) a, D_{1}(i)\right\rangle+\left\langle a \sigma(i), D_{1}(j)\right\rangle \\
& =\left\langle a, D_{1}(i) \sigma(j)+\sigma(i), D_{1}(j\rangle\right.
\end{aligned}
$$

So $D_{1}: I \rightarrow A^{*}$ is a $\sigma$-derivation. Since $A$ has a bounded approximate identity and $\sigma$ is an epimorphism, $A$ is a $\sigma$-neo-unital Banach $I$-module. Thus by [2; Proposition 4.14] $D_{1}$ extends to a $\sigma-$ derivation $\widetilde{D}: A \rightarrow A^{*}$ which extends $D$.

Proposition 14 Let $A$ be a Banach algebra with a bounded approximate identity and $\sigma$ be a bounded idempotent epimorphism of $A$. Let $\varphi$ be a non-trivial character on $A$ and $I=\operatorname{ker} \varphi$ which $\sigma(I) \subseteq I$. Suppose that $D: I \rightarrow I^{*}$ be a $\sigma$-derivation. If $\left(\bar{I}^{2}\right)=I$, then there is at most one extension of $D$ to a $\sigma$ derivation $D: A \rightarrow A^{*}$.

Proof. Let $\widetilde{D}_{1}$ and $\widetilde{D_{2}}$ be two extension of a $\sigma$-derivatin $D$. Then $\widetilde{D}_{1}-D_{2}$ is a $\sigma$-derivation extending the zero derivation. Therefore we assume that $\widetilde{D}$ be any extension of $D=0$. So for each $i, j \in I$ and $a \in A$ we have,

$$
\begin{aligned}
\langle a, \widetilde{D}(i, j\rangle & =\langle a, \sigma(i) \widetilde{D}(j)+\widetilde{D}(i) \sigma(j)\rangle \\
& =\langle a \sigma(i), \widetilde{D}(j)\rangle+\langle\sigma(j) a, \widetilde{D}(i)\rangle \\
& =\langle a \sigma(i), D(j)\rangle+\langle\sigma(j) a, D(i)\rangle=0
\end{aligned}
$$

Since $\left(\overline{I^{2}}\right)=I$, we have $\langle a, \widetilde{D}(i)\rangle=0$ for all $i \in I$ and $a \in A$. Also for each $a, b \in A$ we have,

Thus

$$
\left\langle\sigma(a) \sigma(b), \widetilde{D}\left(e_{\alpha}\right)\right\rangle=\left\langle\sigma(a), \widetilde{D}\left(b e_{\alpha}\right)\right\rangle-\left\langle\sigma\left(e_{\alpha}\right) \sigma(a), \widetilde{D}(b)\right\rangle
$$

$$
\lim _{\alpha}\left\langle\sigma(a) \sigma(b), \widetilde{D}\left(e_{\alpha}\right)\right\rangle=0
$$

As $\sigma$ is an epimorphism and $A$ has ${ }^{\alpha}$ a bounded approximate identity, by the Cohen Factorisation theorem, every element of $A$ can be factorized. Hence

$$
\langle b, \widetilde{D}(a)\rangle=\lim \left\langle b, \widetilde{D}\left(a-\varphi(a) e_{\alpha}\right)\right\rangle=0
$$

because $a-\varphi(a) e_{\alpha} \in I$. Which shows thă any extension of $D$ is unique.

Next, assume that $A$ is a complex Banach space which has dimension at least 2 and let $0 \neq \varphi \in$ Ball $\left(A^{*}\right)$. Define a multiplication on $A$ by

$$
a \cdot b=\varphi(a) b \quad(a, b \in A)
$$

This multiplication evidently makes $A$ into a Banach algebra denoted by $A_{\varphi}$. Which is called the ideally factored algebra associated to $\varphi$. It is easy to see that $A_{\varphi}$ has left identity $e$ which is that element 
in $A$ such that $\varphi(e)=1$, while it has not right approximate identity. Also if $I$ is a proper ideal of $A_{\varphi}$, then $I \subseteq \operatorname{ker} \varphi$. Suppose that $\sigma: A_{\varphi} \rightarrow A_{\varphi}$ be defined by $\sigma(a)=\varphi(a) e$. Then $\sigma$ is the only idempotent endomorphism of $A_{\varphi}$.

Proposition $15 A_{\varphi}$ is $\sigma$-ideally amenable, where $\sigma(a)=\varphi(a) e$ is the only idempotent endomorphism of $A_{\varphi}$.

Proof. Let $I$ be a closed two-sided ideal in $A_{\varphi}$ and $D: A_{\varphi} \rightarrow I^{*}$ be a $\sigma$-derivation. Since $I \subseteq \operatorname{ker} \varphi$, for each $a, b \in A_{\varphi}$ and $i \in I$ we have,

$$
\begin{aligned}
\varphi(a)\langle i, D(b)\rangle & =\langle i, D(a \cdot b)\rangle=\langle i, \sigma(a) \cdot D(b)+D(a) \cdot \sigma(b)\rangle \\
& =\langle i, \sigma(a) \cdot D(b)\rangle+\langle\sigma(b) \cdot i, D(a)\rangle \\
& =\varphi(b)\langle i, D(a)\rangle
\end{aligned}
$$

In (1), set $b=e$. So we have,

$$
\varphi(a)\langle i, D(e)\rangle=\varphi(e)\langle i, D(a)\rangle=\langle i, D(a)\rangle
$$

On the other hand, let $i^{*} \in I^{*}$, and let $\delta_{i^{*}}^{\sigma}: A \rightarrow I^{*}$ be the $\sigma$-inner derivation specified by $i^{*}$. Then for each $a \in A_{\varphi}$ and $i \in I$ we have,

Set $i^{*}=-D(e)$. By (2) , (3) we have

$$
\begin{aligned}
\left\langle i, \delta_{i^{*}}^{\sigma}(a)\right\rangle & =\left\langle i, \sigma(a) \cdot i^{*}-i^{*} \cdot \sigma(a)\right\rangle \\
& =\left\langle i \cdot \sigma(a), i^{*}\right\rangle-\left\langle\sigma(a) \cdot i, i^{*}\right\rangle \\
& =\varphi(i)\left\langle\sigma(a), i^{*}\right\rangle-\varphi(\sigma(a))\left\langle i, i^{*}\right\rangle \\
& =-\varphi(a)\left\langle i, i^{*}\right\rangle
\end{aligned}
$$

$$
\left\langle i, \delta_{i^{*}}^{\sigma}(a)\right\rangle=-\varphi(a)\langle i,-D(e)\rangle=\langle i, D(a)\rangle, \quad(a \in A \varphi, i \in I)
$$

Therefore, $D=\delta_{i^{*}}^{\sigma}$. Thus $A \varphi$ is $\sigma$-ideally amenable.

\section{References}

[1] M. Eshaghi Gordji, T. Yazdanpanah, Derivations into duals of ideals of Banach algebras, Proc. Indian Acad. Sci. 114, 4 (2004), 399-408.

[2] M. Momeni, T. Yazdanpanah, and M.R. Mardanbeigi, $\sigma$-Approximately Contractible Banach Algebras, Abstract and Applied Analysis, Volume 2012(2012), Article ID 653140, 20 pages, doi: $.1155 / 2012 / 653140$.

[3] H.G. Dales, F. Ghahramani and N. Gronbaek, Derivations into iterated duals of Banach algebras, Studia Math. 128 (1998) 19-54.

[4] HE. Dales, Banach algebra and automatic continuity, London Mathematical Society Monographs (Oxford: Clarendon Press) (2000) vol. 24 